\title{
Therapeutic effects of sorafenib on the A549/DDP human lung adenocarcinoma cell line in vitro
}

\author{
XIANG-QI CHEN ${ }^{1,2^{*}}$, YU-LAN WANG ${ }^{1 *}$, ZHI-YING LI ${ }^{1,2^{*}}$ and TING-YAN LIN ${ }^{1,2}$ \\ ${ }^{1}$ Teaching and Research Department of Respiratory Medicine, Union Clinical Medical College of Fujian Medical University; \\ ${ }^{2}$ Department of Respiratory Medicine, Fujian Medical University Union Hospital, \\ Fuzhou, Fujian 350001, P.R. China
}

Received July 22, 2013; Accepted March 4, 2014

DOI: $10.3892 / \mathrm{mmr} .2014 .2163$

\begin{abstract}
The aim of the present study was to observe the effects of sorafenib on the proliferation, apoptosis and invasion of A549/DDP cisplatin-resistant lung adenocarcinoma cells cultured in vitro. The A549/DDP cisplatin-resistant lung adenocarcinoma cell strain was cultured in vitro, the cell culture group incubated in culture medium only was set as the control group (Group S0) and the four concentration gradients of sorafenib were added to the culture groups as the experimental groups: S1, $2 \mu \mathrm{mol} / 1 ; \mathrm{S} 2,4 \mu \mathrm{mol} / 1 ; \mathrm{S} 3,8 \mu \mathrm{mol} / 1$; and $\mathrm{S} 4,16 \mu \mathrm{mol} / \mathrm{l}$. The MTT assay was used to determine the growth inhibition rate of the cells, which were respectively subjected to sorafenib treatment for 24,48 and $72 \mathrm{~h}$. Flow cytometry was used to determine the rate of apoptosis of cells in each group following sorafenib treatment for $72 \mathrm{~h}$. Furthermore, the Transwell invasion experiment was used to determine the effect on A549/DDP cell invasion following sorafenib treatment for $24 \mathrm{~h}$. Based on the MTT assay, it was found that the inhibition rates of A549/DDP cisplatin-resistant lung adenocarcinoma cells in groups S1-4 following sorafenib treatment for $24 \mathrm{~h}$ were $4.58 \pm 2.82,14.93 \pm 2.62,37.58 \pm 7.13$ and $58.39 \pm 8.15 \%$, respectively. For $48 \mathrm{~h}$, inhibition rates in S1-4 were $14.98 \pm 2.93,26.28 \pm 7.31,63.00 \pm 3.05$ and $78.84 \pm 3.96 \%$, respectively, and for $72 \mathrm{~h}$, inhibition rates were $18.80 \pm 2.82$, $32.71 \pm 2.55,75.51 \pm 4.73$ and $87.50 \pm 3.36 \%$, respectively. The difference in the inhibition rates of cells among the experimental groups for the same incubation time showed statistical significance $(\mathrm{P}<0.05)$. Flow cytometric analysis indicated that the rate of apoptosis in the control group was $8.88 \pm 0.81 \%$ following sorafenib treatment for $72 \mathrm{~h}$, and the
\end{abstract}

Correspondence to: Professor Ting-yan Lin, Department of Respiratory Medicine, Fujian Medical University Union Hospital, 29 Xinquan Road, Fuzhou, Fujian 350001, P.R. China

E-mail:drlcxhhx@126.com

*Contributed equally

Key words: sorafenib, lung adenocarcinoma, cell growth, apoptosis, invasion rates of apoptosis in groups S1-4 were, $12.84 \pm 0.24,17.27 \pm 0.78$, $21.98 \pm 0.75$ and $49.67 \pm 1.38 \%$, respectively. The rate of apoptosis in each experimental group was higher compared with that in the control group $(\mathrm{P}<0.05)$. The difference in the rate of apoptosis among the experimental groups was statistically significant $(\mathrm{P}<0.05)$. The Transwell assay showed that the number of cells permeating the septum in the control group was $82.7 \pm 2.3 /$ high power lens (HP), while the average number of cells permeating septum in groups S1-4 following treatment with sorafenib for $24 \mathrm{~h}$ was $58.2 \pm 2.5,41.3 \pm 1.3,22.6 \pm 2.1$ and $14.7 \pm 1.1 / \mathrm{HP}$, which was significantly lower compared with the control group. The number of cells permeating the septum in each experimental group decreased with the enhancement of the concentration gradient. The differences were statistically significant $(\mathrm{P}<0.05)$. In conclusion, sorafenib inhibits the proliferation of A549/DDP cisplatin-resistant lung adenocarcinoma cells in a time- and concentration-dependent manner. In addition, sorafenib induces apoptosis in A549/DDP cisplatin-resistant lung adenocarcinoma cells, thus reducing their invasiveness.

\section{Introduction}

At present, lung cancer is a malignant tumor with the globally highest morbidity and fatality rates. $\sim 80-85 \%$ of lung cancers are non-small-cell lung carcinoma (NSCLC). Surgical resection is considered the main treatment for NSCLC, with surgery being indicated for $20-30 \%$ of patients and the postoperative recurrence and the metastasis rate being $\geq 50 \%$ (1). Therefore, for the lung cancer patients who are unable to undergo surgery and those with postoperative recurrence or metastasis, combinations of platinum-containing agents are the primary chemical treatment to extend the lifetime. However, to date, no effective measures have been established for patients who are subject to such regimen control, and who present with new cancer progression. Sorafenib is a small molecule with activity against neoplasms via multiple biological targets. Sorafenib has a dual function of inhibiting tumor cell proliferation and angiogenesis. At present, the Food and Drug Administration (FDA, Silver Spring, MD, USA) has approved sorafenib for the use in the clinical treatment of kidney and liver cancers $(2,3)$. To date, basic research and clinical trials using sorafenib for the prevention and treatment of lung cancer have made positive 
progress globally $(4,5)$. The present study used the A549/DDP cisplatin-resistant lung adenocarcinoma cell strain to study the biological effects of sorafenib. Effects on proliferation, apoptosis and invasion of the A549/DDP cisplatin-resistant lung adenocarcinoma cell strain was observed, which provided scientific experimental data and a theoretical basis for the application of sorafenib for the subsequent treatment of cisplatin-resistant lung cancer.

\section{Materials and methods}

Cell strains. The A549 lung adenocarcinoma cell strain and the A549/DDP cisplatin-resistant lung adenocarcinoma cell strain were purchased from the tumor cell bank of the Chinese Academy of Sciences (Shanghai, China), with a resistance index of 13 times.

Main reagents. Sorafenib was purchased from Bayer (Leverkusen, Germany), RPMI-1640 culture medium and fetal bovine serum (FBS) were from HyClone (Logan, UT, USA), cisplatin (DDP) from Haosen Pharmacy, Ltd. (Ganzhou, China), MTT and dimethylsulfoxide (DMSO) from Sigma-Aldrich (St. Louis, MO, USA), Annexin V-fluorescein isothiocyanate (FITC)/propidium iodide (PI) apoptosis kit from BioVision (Mountain View, CA, USA) and Matrigel ${ }^{\circledR}$ from BD Biosciences (Franklin Lakes, NJ, USA).

Cell culture. The A549 and A549/DDP cells were cultured in RPMI-1640 medium at $37^{\circ} \mathrm{C}$ with $5 \% \mathrm{CO}_{2}$ and $95 \%$ saturated humidity. To maintain the stable drug-resistance of A549/DDP cells, $2 \mu \mathrm{mol} / 1 \mathrm{DPP}$ was added into the RPMI-1640 with 10\% FBS for joint culture and the cells were cultured in the medium without DDP for one week prior to beginning the trial.

Experimental groups. The experiment involved five groups: S0 and experimental groups S1, S2, S3 and S4. The control group S0 was incubated with the culture medium RPMI-1640 only; S1-4 were incubated with 2, 4, 8 and $16 \mu \mathrm{mol} / 1$ sorafenib, respectively.

MTT assay. Cells were collected at the exponential growth phase, the cell density was adjusted to $3 \times 10^{4} \mathrm{ml}^{-1}$ and $100 \mu \mathrm{l}$ was inoculated into each well on 6 culture plates and 7 repeated wells set for each 96-well plate. One culture plate was removed each day at the same time, MTT reagent $(20 \mu \mathrm{l})$ was added, placed in a $\mathrm{CO}_{2}$ incubator and incubated for $4 \mathrm{~h}$. Following removal of the medium, DMSO $(100 \mu \mathrm{l})$ was added to each well and the plate was placed on an agitator for $10 \mathrm{~min}$ to fully dissolve the crystals. The optical density (OD) of each well was measured at $490 \mathrm{~nm}$ using an ELISA reader (R\&D Systems, Minneapolis, MN, USA). The blank controls (containing medium, MTT and DMSO) and control wells (containing cells, medium, MTT and DMSO) were measured. The formula adopted for the calculation of the inhibition rate of cell proliferation is: Inhibition rate $=100 \% \times[(\mathrm{OD}$ value of the control well $-\mathrm{OD}$ value of the blank control well)-(OD value of the experimental well - OD value of the blank control well)]/(OD value of control well - OD value of the blank control well). Each experiment was repeated three times.
Flow cytometric analysis of apoptosis. The inoculated cells were cultured in a $5 \% \mathrm{CO}_{2}$ incubator for $24 \mathrm{~h}$ and the original culture medium was discarded. Different concentrations of sorafenib $(2,4,8$ and $16 \mu \mathrm{mol} / \mathrm{l})$ were added to the experimental groups at $2 \mathrm{ml} /$ well in RPMI medium. The control group was added with the same amount of culture medium without sorafenib. Three complexes were prepared for each group. The culture plates were incubated for a further $72 \mathrm{~h}$. The cells of each group were digested with EDTA-free pancreatin (Gibco, Grand Island, NY, USA) and collected. Binding buffer (500 $\mu \mathrm{l}$; KeyGen Biotech, Nanjing, China) was added to prepare a cell suspension. Under the exclusion of light, $5 \mu 1$ Annexin V-FITC and $5 \mu \mathrm{l}$ PI was added to each group of cell suspension. The groups were allowed to react for $15 \mathrm{~min}$ at room temperature in the dark. Flow cytometric analysis was performed within one hour and the percentage of apoptotic cells was calculated using CellQuest software, version 3.0 (BD Biosciences).

Transwell assay. A549/DDP cells were collected during the exponential growth phase, the concentration was adjusted to $5 \times 10^{5} \mathrm{ml}^{-1}$ and the cells were inoculated into the upper chamber of a Transwell plate $(6.5 \mathrm{~mm}$; 24-well plate, Costar; Corning, NY, USA) at $200 \mu \mathrm{l} /$ well and three repeated wells were set for each group. Sorafenib was added to the upper chamber with a final concentration of 2, 4,8 and $16 \mu \mathrm{mol} / 1$ for the experimental groups, and no reagent was added for the control group. At the same time, RPMI-1640 (500 $\mu \mathrm{l})$ culture medium containing $10 \%$ FBS was added into the lower chamber and the plate was cultured for a further $24 \mathrm{~h}$. The cells were removed from the Transwell chamber, washed twice with phosphate-buffered saline and the filter membrane was fixed with $4 \%$ paraformaldehyde for $30 \mathrm{~min}$. The filter membrane was subjected to crystal violet staining (Beyotime Institute of Biotechnology, Shanghai, China) for 20 min following air drying and was then rinsed with double distilled water. Five fields of view at upper, lower, left, right and central locations were captured under a light microscope $(\mathrm{x} 400)$. To assess the number of cells migrated through the membrane to the lower chamber surface, the average values were analyzed. The number of migrated cells reflected the level of tumor cell invasiveness. Accordingly, a reduced number of cells permeating the septum indicated marked inhibition of invasion.

Statistical analysis. Values are presented as the mean \pm standard deviation and were determined by the statistical software SPSS 11.0 (SPSS Inc., Chicago, IL, USA). Groups were compared using one-way analysis of variance. Homogeneity of variance was tested by the least significant difference method, abd heterogeneity of variance was tested using Dunnett's $\mathrm{T}$ method. $\mathrm{P}<0.05$ was considered to indicate a statistically significant difference.

\section{Results}

Comparison between the growth curves of A549/DDP cisplatin-resistant lung adenocarcinoma cells and A549 parental cells. Microscopic observation indicated that the adherent growth of cisplatin-resistant lung adenocarcinoma cells A549/DDP was marked and vigorous, indicating a satisfying light refraction of 


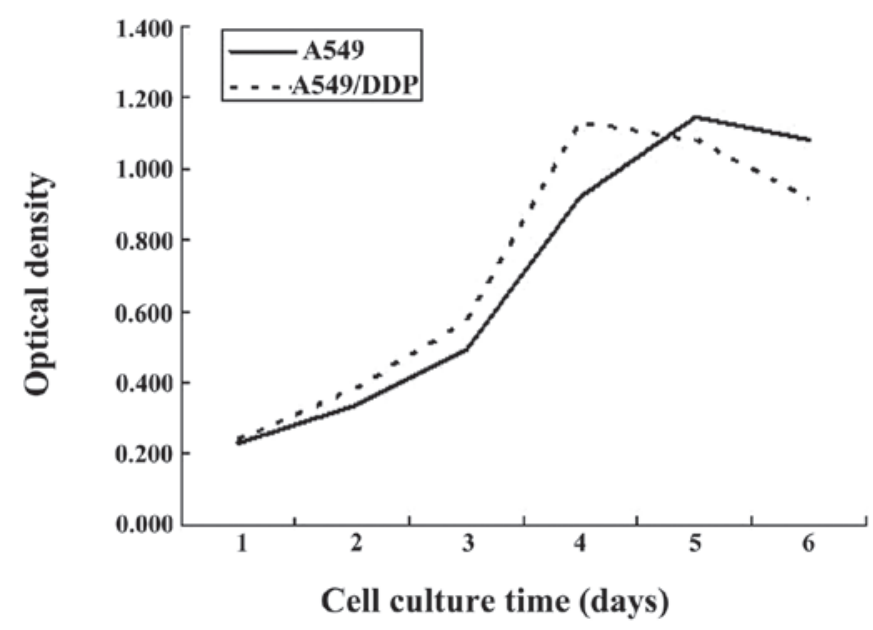

Figure 1. Comparison between the growth curves of A549/DDP cisplatin-resistant lung adenocarcinoma cells and parental A549 cells.

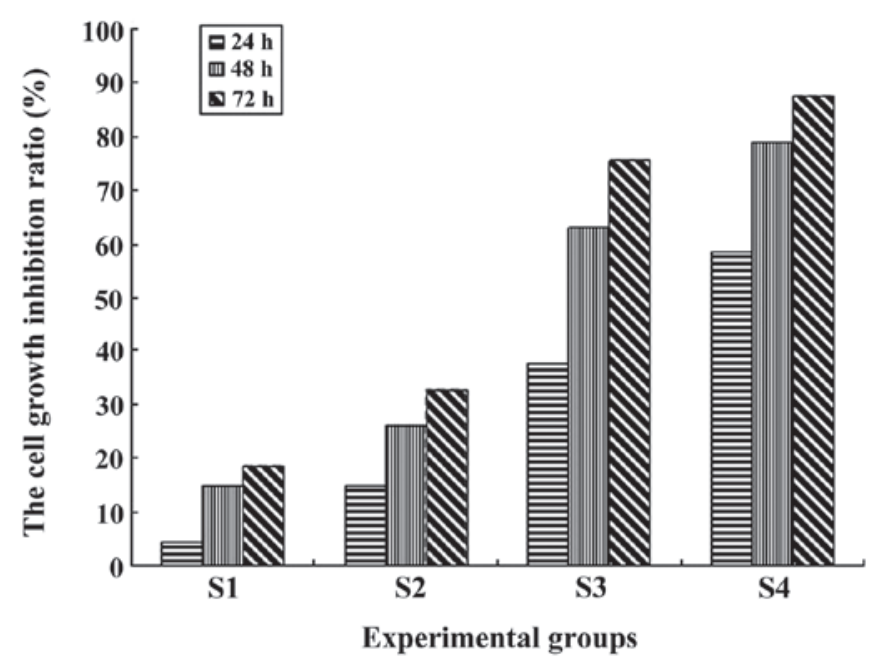

Figure 2. Concentration-dependent growth inhibition by sorafenib on A549/DDP cisplatin-resistant lung adenocarcinoma cells. S1-4, incubation with sorafenib at $2,4,8$ and $16 \mu \mathrm{mol} / 1$.

cytoplasm. Compared with A549 cells, the A549/DDP cells were slightly larger in volume, growing in fusiform or polygonal structures, with a large cell nucleus. Compared with parental A549 cells, the A549/DDP cisplatin-resistant cells exhibited a higher cell proliferation rate and reached the exponential growth phase 2-4 days following passage, while the A549 cells required 3-5 days to reach the exponential growth phase (Fig. 1).

Sorafenib inhibits the proliferation of A549/DDP cisplatin-resistant lung adenocarcinoma cells. The MTT assay was used to determine the inhibition rate of sorafenib on the proliferation of A549/DDP cisplatin-resistant lung adenocarcinoma cells at different concentrations and time-points. The results showed that, compared with the control group, sorafenib inhibited the proliferation of A549/DDP cisplatin-resistant lung adenocarcinoma cells within the concentration range of 2, 4, 8 and $16 \mu \mathrm{mol} / \mathrm{l}$. With the extension of the action time and increase of the concentration, the inhibition rate of sorafenib on A549/DDP cisplatin-resistant lung adenocarcinoma cells

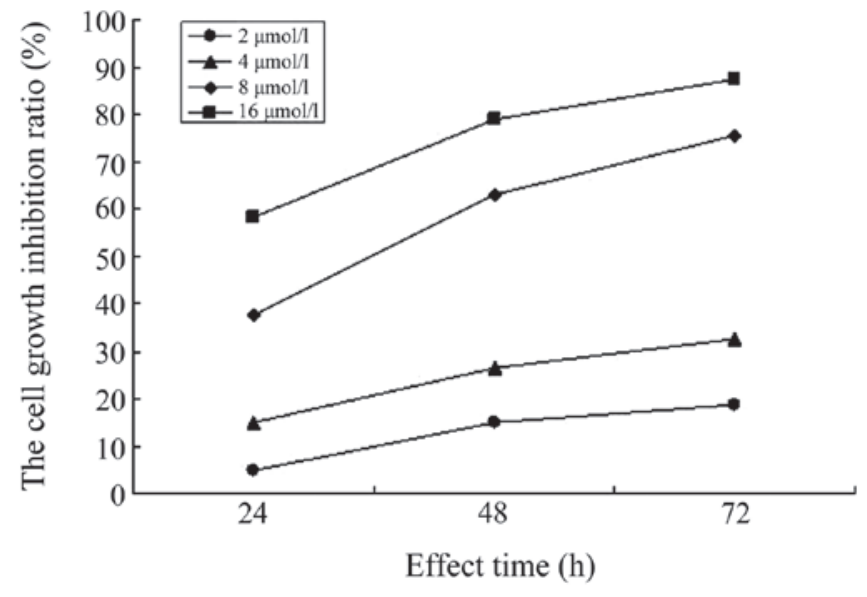

Figure 3. Time-effect curves of the growth inhibition of A549/DDP cells treated with different concentrations of sorafenib. S1-4, incubation with sorafenib at $2,4,8$ and $16 \mu \mathrm{mol} / 1$.

Table I. The inhibition rate of sorafenib on A549/DDP cisplatin-resistant lung adenocarcinoma cells at different incubation times (mean \pm standard deviation).

\begin{tabular}{lrcc}
\hline Group & $24 \mathrm{~h}$ & $48 \mathrm{~h}$ & $72 \mathrm{~h}$ \\
\hline S1 & $4.58 \pm 2.81$ & $14.98 \pm 2.93^{\mathrm{a}}$ & $18.80 \pm 2.82^{\mathrm{b}}$ \\
S2 & $14.93 \pm 2.62$ & $26.28 \pm 7.31^{\mathrm{a}}$ & $32.71 \pm 2.55^{\mathrm{b}}$ \\
S3 & $37.58 \pm 7.13$ & $63.00 \pm 3.05^{\mathrm{a}}$ & $75.51 \pm 4.73^{\mathrm{c}}$ \\
S4 & $58.39 \pm 8.15$ & $78.84 \pm 3.96^{\mathrm{a}}$ & $87.50 \pm 3.36^{\mathrm{c}}$ \\
\hline
\end{tabular}

${ }^{\mathrm{a}} \mathrm{P}<0.01$ vs. $24 \mathrm{~h}, ;{ }^{\mathrm{b}} \mathrm{P}<0.05$ vs. $48 \mathrm{~h} ;{ }^{\mathrm{c}} \mathrm{P}<0.01$ vs. 48 h. S1-4, incubation with sorafenib at $2,4,8$ and $16 \mu \mathrm{mol} / 1$

gradually increased $(\mathrm{P}<0.05)$. In addition, the differences in the inhibition rates of sorafenib on A549/DDP cisplatin-resistant lung adenocarcinoma cells at different times and concentrations were statistically significant $(\mathrm{P}<0.05)$, as shown in Table I and Figs. 2 and 3.

Sorafenib induces apoptosis of A549/DDP cisplatin-resistant lung adenocarcinoma cells. The A549/DDP cisplatin-resistant lung adenocarcinoma cells in the experimental groups S1-4 were respectively treated with sorafenib at the concentrations of 2, 4, 8 and $16 \mu \mathrm{mol} / 1$ for $72 \mathrm{~h}$, and the S0 control group was cultured with RPMI-1640 medium only for $72 \mathrm{~h}$. The rate of apoptosis was determined using Annexin V-FITC/PI staining and flow cytometric analysis. It was observed that the rate of apoptosis of the control group S0 was $8.89 \pm 0.81 \%$ and the rates of apoptosis in the experimental groups S1-4 were $12.84 \pm 0.24,17.27 \pm 0.78,21.98 \pm 0.75$ and $49.67 \pm 1.38 \%$, respectively. Compared with the control group, the differences were statistically significant $(\mathrm{P}<0.01)$; the differences of further pairwise comparison in the same group were also statistically significant $(\mathrm{P}<0.01)$, as shown in Fig. 4.

Sorafenib inhibits the invasion of A549/DDP cisplatin-resistant lung adenocarcinoma cells. The Transwell assay was used to determine the number of cells permeating the 


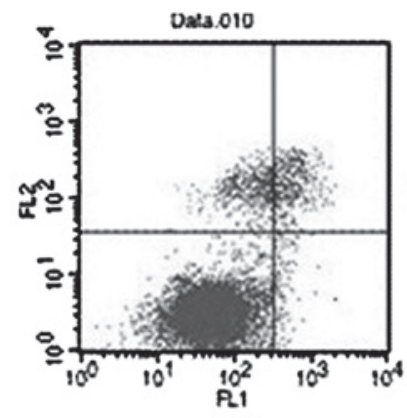

So

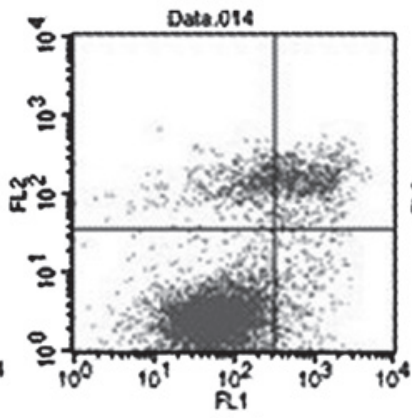

S1

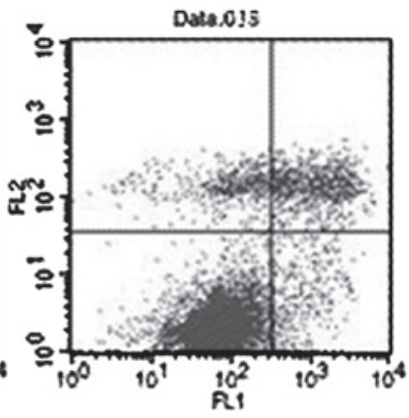

S2

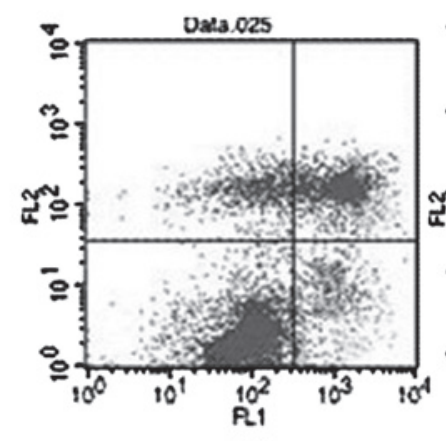

S3

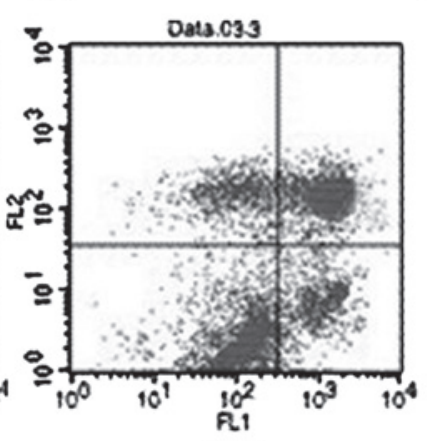

S4

Figure 4. Flow cytometric assessment of apoptosis of A549/DDP cisplatin-resistant lung adenocarcinoma cells following $72 \mathrm{~h}$ sorafenib treatment and staining with Annexin V-fluorescein isothiocyanate/propidium iodide. S0-4, incubation with sorafenib at 0, 2, 4, 8 and 16 $\mu$ mol/1. X-axis represents Annexin V-FITC staining and y-axis represents PI staining. Upper left: necrosis cells; upper right: late apoptotic cells; lower left: normal cells; lower right: early apoptotic cells.

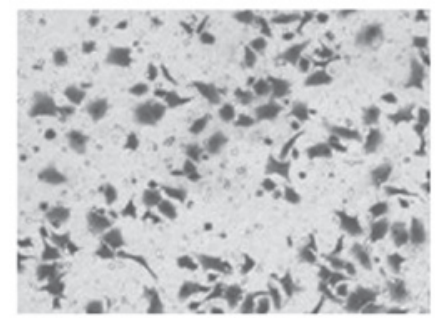

S0

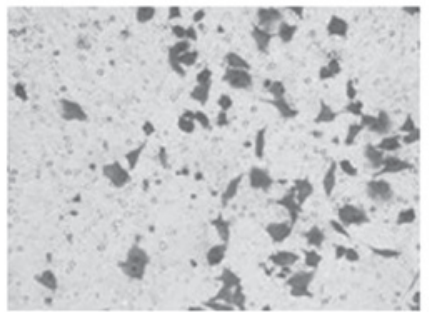

S1

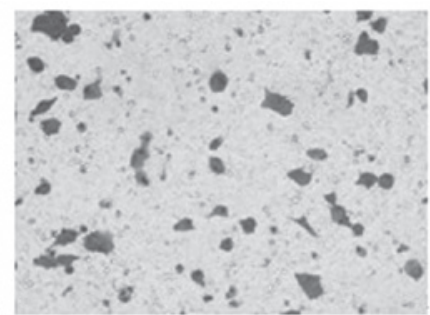

S2

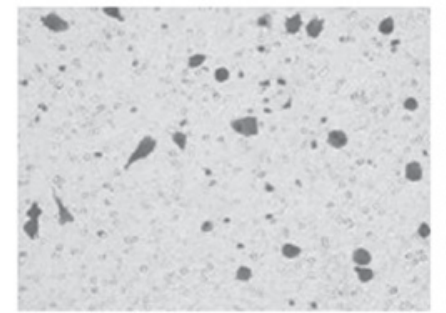

S3

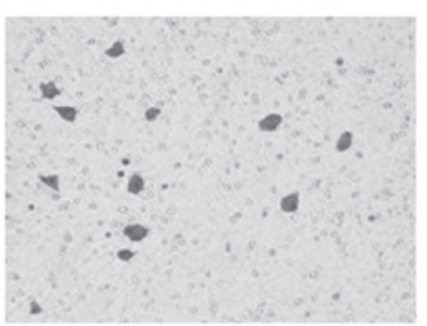

S4

Figure 5. Sorafenib inhibits the invasion of A549/DDP cisplatin-resistant lung adenocarcinoma cells following incubation for 24 h (S0-4, incubation with sorafenib at $0,2,4,8$ and $16 \mu \mathrm{M} / 1$ ). Photomicrographs (magnification, $\mathrm{x} 400$ ) of lower Transwell membranes staned with crystal violet.

Transwell membrane in each group. The results showed that, following $24 \mathrm{~h}$ of incubation, the average number of cells permeating the septum in the normal control group S0 reached $82.7 \pm 2.3 /$ high power lens $(\mathrm{HP})$, while the average number of cells permeating the septum in $\mathrm{S} 1-4$ in the presence of sorafenib at concentrations of 2, 4, 8 and $16 \mu \mathrm{mol} / 1$, were decreased to $58.2 \pm 2.5,41.3 \pm 1.3,22.6 \pm 2.1$ and $14.7 \pm 1.1 / \mathrm{HP}$, respectively. Compared with the control group, the difference in the number of cells permeating the Matrige ${ }^{\circledR}$ of each group was statistically significant $(\mathrm{P}<0.01)$. When the concentration increased and the cell permeation of the septum gradually decreased, the differences obtained by pairwise comparisons among the groups also became statistically significant $(\mathrm{P}<0.01)$. In addition, observation by optical microscopy following crystal violet staining indicated that the cells in the normal control group which had not been treated with sorafenib were of fusiform, polygonal or oval shaped, with a larger cell volume a dense distribution at the lower surface 
of the Transwell chamber, while the cells of the experimental groups, which had been treated with sorafenib, gradually became smaller, pseudopodia decreased and the shape of the cells became round and sparsely scattered at the lower surface of the Transwell chamber (Fig. 5).

\section{Discussion}

Sorafenib is an antineoplastic agent with multiple biological targets, which inhibits tumor growth, invasion and metastasis by preventing the proliferation of tumor cells and angiogenesis in tumor tissue. In 2005 and 2007, the FDA approved the use of sorafenib for the clinical treatment of advanced kidney and liver cancer (6,7). At present, basic research and clinical trials using sorafenib for the prevention and treatment of lung cancer are progressing with positive preliminary results $(4,5)$. Dy et al (8) have tested sorafenib in a Phase II clinical trial as a first-line treatment of advanced NSCLC and the results showed that among the 25 patients selected for the study, three went into partial remission and six achieved a stable condition, with progression-free survival (PFS) for 2.8 months, and a certain clinical efficacy was obtained. Spigel et al (9) performed a clinical trial of combining sorafenib with erlotinib, or using only erlotinib as the second-line treatment of patients with advanced NSCLC. A total of 168 patients were involved. The results showed that the disease control rates of the combination group and the group with only erlotinib were 54 and $38 \%$, respectively, and PFSs were 3.38 and 1.94, respectively, indicating that sorafenib has potential as a second-line treatment. In the present study, in order to provide a novel method for the second-line treatment of NSCLC, the A549/DDP cisplatin-resistant lung adenocarcinoma cell strain was chosen to assess the effect of sorafenib on the proliferation, apoptosis and invasion in vitro.

In the present study, the MTT assay was used to determine the activity of sorafenib on the proliferation of A549/DDP cisplatin-resistant lung adenocarcinoma cells and to observe its inhibition effects. The results showed that compared with the parental A549 cells, the A549/DDP cisplatin-resistant lung adenocarcinoma cells were slightly larger in volume, growing in fusiform or polygonal structures, with a large intracellular nucleus. The cell proliferation was faster, reaching the exponential phase within 2-4 days following passage, while A549 cells reached the exponential phase within 3-5 days following passage, indicating that the metabolic activity of A549/DDP cisplatin-resistant lung adenocarcinoma cells was significantly enhanced and marked mitosis was observed following acquisition of drug-resistance characteristics; thus, they were able to enter the logarithmic phase faster than the parental A549 cells. This is consistent with the observations on the growth characteristics of A549/DDP cisplatin-resistant lung adenocarcinoma cells reported by Giard et al (10). A further analysis of the inhibitory effect of sorafenib on the proliferation of A549/DDP cisplatin-resistant lung adenocarcinoma cells was conducted, showing that sorafenib is capable of inhibiting the proliferation of the A549/DDP cisplatin-resistant lung adenocarcinoma cells within the concentration range of 2-16 $\mu \mathrm{mol} / 1$. Furthermore, following treatment of A549/DDP cells with varying concentrations of sorafenib, the inhibition rate of cells was gradually increased with increasing time, while at the same time, the inhibition rate was gradually increased as well with increasing drug concentration. The results of this trial showed that sorafenib was capable of inhibiting the proliferation of A549/DDP cisplatin-resistant lung adenocarcinoma cells and this inhibitory effect was time- and concentration-dependent. The Annexin V-FITC/PI assay was used to determine the rate of apoptosis of A549/DDP cisplatin-resistant lung adenocarcinoma cells and to observe the induction effect of sorafenib on the apoptosis of A549/DDP cells. The results showed that marked apoptosis appeared in the cells in each experimental group following treatment with sorafenib at different concentrations for $72 \mathrm{~h}$. In addition, with the increase of the drug concentration, the rate of apoptosis also increased. The difference from the control group was statistically significant $(\mathrm{P}<0.05)$, indicating that sorafenib is capable of promoting the apoptosis of A549/DDP cisplatin-resistant lung adenocarcinoma cells, and this effect was dependent on the concentration. The difference obtained by pairwise comparison among experimental groups was also statistically significant $(\mathrm{P}<0.05)$. The results of the present study were similar to those investigating the effects of sorafenib on the proliferation and apoptosis of parental A549 cells reported by Yu et al (11) and Mao et al (12). In the present study, to further determine the influence of sorafenib on the invasiveness of A549/DDP cisplatin-resistant lung adenocarcinoma cells, the Transwell assay was used for each experimental group in the presence of sorafenib for $24 \mathrm{~h}$. The results showed that with increasing drug concentration, the number of cells permeating the basement membrane in each experimental group significantly decreased. The difference between each experimental and the control group was statistically significant $(\mathrm{P}<0.05)$, indicating that sorafenib had an in vitro inhibitory effect on the invasion of A549/DDP cisplatin-resistant lung adenocarcinoma cells. At the same time, it was noted that the cells permeating the septum in each experimental group markedly changed their shape, the occurrence of pseudopodia decreased and the cell volume decreased.

In conclusion, sorafenib inhibited the proliferation of A549/DDP cisplatin-resistant lung adenocarcinoma cells in a time- and concentration-dependent manner. Sorafenib also induces apoptosis and reduces the invasiveness of A549/DDP cells, which provided scientific experimental evidence for the theoretical basis of the application of sorafenib in the subsequent treatment of cisplatin-resistant lung cancer and offers a novel and effective strategy for further treatment of advanced lung cancer (13-15).

\section{References}

1. Lee MW, Kim DS, Min NY and Kim HT: Akt1 inhibition by RNA interference sensitizes human non-small cell lung cancer cells to cisplatin. Int J Cancer 122: 2380-2384, 2008.

2. Yuen JS, Sim MY, Siml HG, et al: Inhibition of angiogenic and non-angiogenic targets by sorafenib in renal cell carcinoma (RCC) in a RCC xenograft model. Br J Cancer 104: 941-947, 2011.

3. Huynh H, Ngo VC, Koong HN, et al: Sorafenib and rapamycin induce growth suppression in mouse models of hepatocellular carcinoma. J Cell Mol Med 13: 2673-2683, 2009.

4. Smit EF, Dingemans AM, Thunnissen FB, et al: Sorafenib in patients with advanced non-small cell lung cancer that harbor K-ras mutations: a brief report. J Thorac Oncol 5: 719-720, 2010.

5. Okamoto I, Miyazaki M, Morinaga R, et al: Phase I clinical and pharmacokinetic study of sorafenib in combination with carboplatin and paclitaxel in patients with advanced non-small cell lung cancer. Invest New Drugs 28: 844-853, 2010. 
6. Kim S, Yazici YD, Calzada G, et al: Sorafenib inhibits the angiogenesis and growth of orthotopic anaplastic thyroid carcinoma xenografts in nude mice. Mol Cancer Ther 6: 1785-1792, 2007.

7. Escudier B, Eisen T, Stadler WM, et al: Sorafenib for treatment of renal cell carcinoma: final efficacy and safety results of the phase III treatment approaches in renal cancer global evaluation trial. J Clin Oncol 27: 3312-3318, 2009.

8. Dy GK, Hillman SL, Rowland KM Jr, et al: A front-line window of opportunity phase 2 study of sorafenib in patients with advanced nonsmall cell lung cancer: North Central Cancer Treatment Group Study N0326. Cancer 116: 5686-5693, 2010.

9. Spigel DR, Burris HA 3rd, Greco FA, et al: Randomized, double-blind, placebo-controlled, phase II trial of sorafenib and erlotinib or erlotinib alone in previously treated advanced non-small-cell lung cancer. J Clin Oncol 29: 2582-2589, 2011.

10. Giard DJ, Aaronson SA, Todaro GJ, et al: In vitro cultivation of human tumors: establishment of cell lines derived from a series of solid tumors. Natl Cancer Inst 51: 1417-1423, 1973.
11. Yu C, Friday BB, Lai JP, et al: Cytotoxic synergy between the multikinase inhibitor sorafenib and the proteasome inhibitor bortezomib in vitro: induction of apoptosis through Akt and c-Jun NH2-terminal kinase pathways. Mol Cancer Ther 5: 2378-2387, 2006.

12. Mao WF, Shao MH, Gao PT, et al: The important roles of RET, VEGFR2 and the RAF/MEK/ERK pathway in cancer treatment with sorafenib. Acta Pharmacol Sin 33: 1311-1318, 2012.

13. Carter CA, Chen C, Brink C, et al: Sorafenib is efficacious and tolerated in combination with cytotoxic or cytostatic agents in preclinical models of human non-small cell lung carcinoma. Cancer Chemother Pharmacol 59: 183-195, 2007.

14. Li J, Pan YY and Zhang Y: Sorafenib combined with gemcitabine in EGFR-TKI-resistant human lung cancer cells. Oncol Lett 5: 68-72, 2013.

15. Giovannetti E, Labots M, Dekker H, et al: Molecular mechanisms and modulation of key pathways underlying the synergistic interaction of sorafenib with erlotinib in non-small-cell-lung cancer (NSCLC) cells. Curr Pharm Des 19: 927-939, 2013. 